\title{
Cell Cycle-Mediated Cardiac Regeneration in the Mouse Heart
}

Arash Eghbali ${ }^{1}$, Austin Dukes ${ }^{1}$, Karl Toischer ${ }^{2}$, Gerd Hasenfuss ${ }^{2}$ and Loren J. Field ${ }^{1}$

(1) Krannert Institute of Cardiology and Wells Center for Pediatric Research, Indiana University

School of Medicine, Indianapolis, USA, and (2) Department of Cardiology and Pneumology,

Heart Center, Georg-August-University, Goettingen, Germany and DZHK (German Center for

Cardiovascular Research) Partnersite Goettingen

\section{Corresponding author:}

Loren J. Field

Krannert Institute of Cardiology and the Herman B Wells Center for Pediatric Research

Indiana University School of Medicine

1044 W. Walnut Street

Indianapolis, IN 46202, USA

Tel.: +1-317-274-5085

Fax: +1-317-278-9298

lifield@iu.edu

\section{Authors:}

Arash Eghbali

aeghbali@iu.edu

Austin Dukes

audukes@iu.edu

This is the author's manuscript of the article published in final edited form as:

Eghbali, A., Dukes, A., Toischer, K., Hasenfuss, G., \& Field, L. J. (2019). Cell Cycle-Mediated Cardiac Regeneration in the Mouse Heart. Current Cardiology Reports, 21(10), 131. https://doi.org/10.1007/s11886-019-1206-9 
Karl Toischer

ktoischer@med.uni-goettingen.de

Gerd Hasenfuss

hasenfus@med.uni-goettingen.de

Loren J. Field

lifield@iu.edu

\begin{abstract}
Many forms of heart disease result in the essentially irreversible loss of cardiomyocytes. The ability to promote cardiomyocyte renewal may be a promising approach to reverse injury in diseased hearts.

Purpose of Review: To describe the impact of cardiomyocyte cell cycle activation on cardiac function and structure in several different models of myocardial disease.

Recent Findings: Transgenic mice expressing cyclin D2 (D2 mice) exhibit sustained cardiomyocyte renewal in the adult heart. Earlier studies demonstrated that D2 mice exhibited progressive myocardial regeneration in experimental models of myocardial infarction, and that cardiac function was normalized to values seen in sham operated litter mates by 180 days post-injury. D2 mice also exhibited markedly improved atrial structure in a genetic model of atrial fibrosis. More recent studies revealed that D2 mice were remarkably resistant to heart failure induced by chronic elevated afterload as compared to their wild type (WT siblings), with a 6-fold increase in median survival as well as retention of relatively normal cardiac function. Finally, D2 mice exhibited a progressive recovery in cardiac function to normal levels and a
\end{abstract}


concomitant reduction in adverse myocardial remodeling in an anthracycline cardiotoxicity model.

Summary: The studies reviewed here make a strong case for the potential utility of inducing cardiomyocyte renewal as a means to treat injured hearts. Several challenges which must be met to develop a viable therapeutic intervention based on these observations are discussed.

Keywords: cardiac regeneration; cardiomyocyte renewal; cell cycle regulation

\section{Introduction}

It is largely believed that cardiomyocyte renewal in the adult mammalian heart occurs via proliferation of pre-existing cardiomyocytes [1, 2], as opposed to the activity of cardiomyogenic stem cells [3, 4]. The intrinsic renewal capacity of adult mammalian cardiomyocytes is quite limited, with similarly low rates reported for rodents and humans [5-8]. Consequently, injuries causing cardiomyocyte death, as for example myocardial infarction, result in reparative fibrosis as opposed to overt tissue regeneration [9]. Fibrosis results in stiffening of the myocardial wall, which in turn increases cardiomyocyte stress leading to additional cell loss, additional fibrosis and ultimately pump failure. Treatments have traditionally relied on interventions aimed at restoring perfusion following acute ischemic events, interventions aimed at reducing the vulnerability of at-risk myocardium (as for example blocking apoptosis or enhancing angiogenesis), interventions aimed at enhancing contractility of the surviving myocardium, and interventions aimed at modulating fibrosis. Advances in our understanding of the molecular regulation of cell cycle activity suggest an additional approach, namely promoting myocardial regeneration by activating cardiomyocyte renewal. This review 
will focus on a model developed in our laboratory which exhibits increased rates of cardiomyocyte cell cycle activity in the adult myocardium. These animals exhibit regenerative cardiac growth, with a concomitant improvement in both cardiac architecture and function following cardiac injury in a variety of experimental models.

\section{Cyclin D2 expression results in sustained cardiomyocyte cell cycle activity in adult mice}

The notion of inducing cardiomyocyte cell cycle with the hope of driving regeneration of the myocardium is not new. Early studies relied on stimulating cell cycle regulatory pathways in cultured cardiomyocytes via pharmacologic or genetic intervention [10]. The advent of technologies to generate genetically modified animal models harboring either gain-of-function or loss-of-functions alterations facilitated studies demonstrating that relatively simple genetic changes can result in profound increases in cardiomyocyte cell cycle activity. For example, early experiments demonstrated that expression of a powerful cell cycle regulatory molecule, the SV40 Large T Antigen protein, was sufficient to induce proliferation of atrial $[11,12]$ or ventricular [13] cardiomyocytes. Subsequently, numerous genetic modifications have been shown to impact cardiomyocyte cell cycle activity to varying degrees.

One such model utilized the alpha cardiac myosin heavy chain transcriptional regulatory sequences to target expression of sequences encoding cyclin D2 (Figure 1A, upper panel shows a schematic diagram of the transgene) [14]. Cyclin D2 (subsequently designated CCND2) is a cofactor which regulates the activity of cyclin-dependent kinase 4 (CDK4), which mediates transit through the restriction point of the cell cycle. Targeted expression of cyclin D2 in adult cardiomyocytes was sufficient to induce expression of endogenous CDK4 (Figure 1A, lower left panels). Adult transgenic mice (designated D2 mice) exhibited an approximately 400- 
fold increase in the level of ventricular cardiomyocyte S-phase activity as compared to their wildtype (WT) siblings, as determined by tritiated thymidine incorporation using a transgenic reporter to identify cardiomyocyte nuclei (Figure 1A, lower right panel; thymidine incorporation levels were roughly $0.0005 \%$ vs. $0.2 \%$ for WT vs. D2 mice, respectively, following a single injection of isotope).

\section{Cell cycle activity restores ventricular cardiac structure and function following myocardial infarction}

To determine the impact of cardiomyocyte cell cycle activity following ischemic injury to the heart, D2 mice and their WT siblings were subjected to permanent coronary artery ligation [15]. WT hearts examined at 7 days or 180 days post-infarction exhibited transmural defects from the apex to the base (Figure 1B, left; Azan staining, grey indicates scar while magenta indicates viable myocardium). D2 hearts harvested at 7 days post-infarction exhibited a similar transmural defect as observed in WT mice, indicating that cyclin D2 expression did not bestow any cardioprotective activity to acute injury. At 180 days post-infarction, D2 hearts exhibited myocardial tissue along the endocardial and epicardial surface of the scar, from the apex to the base (Figure 1B, right, see arrows). Because these structures were not apparent in hearts at 7 days post-infarction, their presence is indicative of de novo myocardial growth.

To determine the impact of cardiomyocyte cell cycle activity on cardiac function, D2 mice and their WT siblings underwent permanent coronary artery ligation or sham surgery, and were then subjected to hemodynamic analyses using intracardiac pressure-volume catheters.

$\mathrm{dP} / \mathrm{dt}_{\max } / \mathrm{EDV}$, a functional parameter which is not influenced by differences in heart rate, was similarly suppressed in infarcted WT and D2 mice as compared to their sham-operated controls (Figure 1C; $y$-axis indicates the percentage normalized to the sham-operated 
animals), in agreement with the similar degree of myocardial injury observed via histologic analysis. Cardiac function remained suppressed in the infarcted WT mice at later time points, but progressively improved in the infarcted D2 mice. By 180 days post-infarction, cardiac function in infarcted D2 mice was not statistically different than that observed in the shamoperated controls. Thus, cardiomyocyte cell cycle activation can promote both structural and functional improvement following myocardial infarction.

\section{Cell cycle activity restores atrial myocardial structure in a genetic model of fibrosis}

Transforming growth factor (TGF)- $\beta 1$ is a cytokine which can regulate numerous biological activities. Increased TGF- $\beta 1$ expression is observed following cardiac injuries which result in increased myocardial fibrosis. Interestingly, transgenic mice expressing a constitutively active form of TGF- $\beta 1$ in cardiomyocytes (TGF mice) exhibit atrial but not ventricular fibrosis (Figure 1D, left panel; red indicates collagen while green indicates viable myocardium) [16]. Atrial fibrosis in this model, which was more pronounced in the right atria, resulted from TGF- $\beta 1$-induced cardiomyocyte apoptosis. To determine the impact of cardiomyocyte cell cycle activity on atrial fibrosis in this model, TGF and D2 mice were intercrossed and the resulting animals analyzed at 12 weeks of age [17]. As expected, right atrial cardiomyocyte cell cycle activity was undetectable in the TGF mice; in contrast, tritiated thymidine positive right atrial cardiomyocytes were detected in TGF / D2 double transgenic mice ( $1.0 \pm 0.3$ cells per section). Cardiomyocyte cell cycle activity in the TGF / D2 double transgenic animals was accompanied by a marked reduction in right atrial fibrosis as compared to mice carrying only the TGF transgene (Figure 1D); interestingly, similar levels of myocyte apoptosis were seen in TGF vs TGF / D2 mice, as measured by activated caspase 3 immune reactivity $(0.6 \pm 0.31$ cells per section vs. $0.83 \pm 0.14$ cells per section, respectively). 
Thus, cardiomyocyte cell cycle activity can antagonize fibrosis secondary to cytokine-induced atrial cardiomyocyte death.

\section{Cell cycle activity blocks progression to heart failure in a chronic pressure overload model}

Chronically elevated afterload (as occurs in hypertension, aortic stenosis or valvular disease) induces concentric cardiac hypertrophy, and over time, adverse myocardial remodeling and pump failure. Transverse aortic constriction (TAC) is frequently used to model chronically elevated afterload in experimental animals. To determine the impact of cardiomyocyte cell cycle activity on the progression of heart failure in response to elevated afterload, D2 mice and their WT littermates were subjected to TAC surgery [18]. WT mice rapidly succumbed to TAC, with $50 \%$ of the animals dying within 50 days of surgery, and all animals dying within 210 days (Figure 2A). Cardiomyocyte cell cycle activity in D2 mice resulted in a 6-fold increase in median survival following TAC, with some mice surviving for as long as 450 days post-surgery. Echocardiography revealed that by 10 weeks post-TAC surgery, WT mice exhibited decreased left ventricular fractional shortening (Figure 2B) which was accompanied with diastolic dilation, both hallmarks for the presence of heart failure. In contrast, cardiac function and chamber volume was normal in D2 mice at 10 weeks post-TAC, consistent with the markedly enhanced survival of these animals.

Cardiomyocyte phosphorylated histone $\mathrm{H} 3(\mathrm{p}-\mathrm{H} 3)$ immune reactivity was quantitated to determine the degree of cell cycle progression (once again a reporter transgene was used to identify cardiomyocyte nuclei). Relatively high rates of cardiomyocyte $\mathrm{p}-\mathrm{H} 3$ immune reactivity were seen in the ventricles of D2 sham mice, consistent with the elevated levels of tritiated thymidine incorporation described above (note that the temporal duration of S-phase is 
approximately 20 -fold longer than M-phase, accounting for the difference in the absolute levels of tritiated thymidine incorporation vs. p-H3 immune reactivity). Interestingly, an almost 3-fold increase in $\mathrm{p}-\mathrm{H} 3$ immune reactivity was seen in D2 TAC mice, indicating that the injury enhanced cell cycle activity in this model. Analysis of dispersed cell preparations revealed that more cardiomyocytes were present in D2 sham hearts as compared to WT sham hearts when analyzed at 10 weeks post-surgery, consistent with the presence of sustained cell cycle activity in the D2 hearts (Figure 2D). Moreover, more cardiomyocytes were present in D2 TAC hearts as compared to the D2 sham hearts, consistent with the injury-induced increase in $\mathrm{p}-\mathrm{H} 3$ levels. Reduced levels of cardiomyocyte apoptosis (Figure 2E) and reduced levels of myocardial fibrosis (Figure 2F) were observed in D2 TAC hearts as compared to WT TAC hearts. Thus, cardiomyocyte cell cycle activity can reduce adverse remodeling and antagonize heart failure progression in response to chronically elevated afterload.

\section{Cell cycle activity restores cardiac function in a juvenile anthracycline cardiotoxicity model}

Although anthracyclines like doxorubicin, daunomycin, epirubicin and idarubicin are potent anti-cancer chemotherapeutics, their use is limited due to rather severe cumulative dose-dependent cardiotoxic side effects. Juvenile patients are particularly sensitive to anthracycline-induced cardiotoxicity. To determine the impact of cardiomyocyte cell cycle activity on anthracycline-induced cardiotoxicity, juvenile (2 week old) D2 mice and their WT littermates received 5 weekly injections of doxorubicin (DOX) or saline, and cardiac function was interrogated via echocardiography at 1,9 and 13 weeks after the last DOX treatment (corresponding to 7, 15 and 19 weeks of age) [19]. Cardiac function was similar and stable in saline-treated WT and D2 mice (Figure 3A). DOX treatment resulted in a marked decrease in 
cardiac function in WT mice at 1 week after the last DOX treatment, and function remained suppressed over the duration of the study. Cardiac function was also decreased in D2 mice at 1 week after the last DOX treatment, but function progressively improved over the course of the study and was indistinguishable from saline treated animals by 13 weeks after the last DOX injection.

Histologic analyses were performed at 1 week (designated the acute stage) and 13 weeks (designated the late stage) after the last DOX treatment. Cardiomyocyte apoptosis was quantitated via activated caspase 3 immune reactivity, and cardiomyocyte cell cycle activity was quantitated via tritiated thymidine incorporation. To facilitate comparison of cardiomyocyte drop-out and cardiomyocyte renewal, the data was normalized to events per $\mathrm{mm}^{2}$ (Figure 3B). DOX treatment of WT mice resulted in cardiomyocyte apoptosis during the acute stage, which increased by approximately 3-fold in late stage hearts, in agreement with previous studies using this juvenile cardiotoxicity model [20]. Essentially no cardiomyocyte cell cycle activity was detected in DOX-treated WT hearts in the acute or late stage. Although DOX treatment resulted in a similar level of cardiomyocyte apoptosis in acute stage D2 hearts, no increases were observed in late stage D2 DOX-treated hearts. As expected, cardiomyocyte cell cycle activity was observed in DOX-treated D2 hearts in both the acute and late stage. Of note, the number of cell cycle events per $\mathrm{mm}^{2}$ was greater than the number of apoptotic events per $\mathrm{mm}^{2}$ in the D2 DOX-treated hearts in both the acute and late stages, suggesting a trend toward myocardial regeneration despite the presence of on-going cardiomyocyte apoptosis. Consistent with this, myocardial fibrosis was markedly reduced in late stage DOX-treated D2 hearts as compared to late stage DOX-treated WT hearts (Figure 3C). Thus, cardiomyocyte 
cell cycle activity can antagonize adverse remodeling and restore cardiac function following anthracycline-induced cardiotoxicity.

\section{Future considerations}

The studies reviewed above indicate that targeted expression of cyclin D2 is sufficient to drive cardiomyocyte proliferation, and more importantly, that the presence of cardiomyocyte cell cycle activity has a positive impact on myocardial function and/or structure in a variety of injury models. The observation of progressive functional and structural improvement over the long-term (i.e., 6 months post-infarct, 10 weeks post-TAC, and 13 weeks post-cardiotoxic drug exposure), supports the notion that recovery results from the presence of active proliferation as opposed to transgene-mediated cardioprotective activity. Indeed, when considering the magnitude of the cell cycle response in D2 mice, one would a priory predict that a long-term follow-up would be required to detect a beneficial effect with this model. A caveat of the D2 model is that transgene expression in the ventricle is activated during the temporal window of cardiomyocyte cell cycle withdrawal which occurs in early postnatal life (i.e., expression may activate prior to cardiomyocyte terminal differentiation). However, unpublished studies from our group demonstrate that de novo activation of cyclin D2 in adult cardiomyocytes using a tetracycline-regulated conditional system gives rise to a similar induction of cell cycle activity. Moreover, de novo expression of a number of other genes has been shown to activate cardiomyocyte cell cycle activity in adults. Thus, there is reasonable evidence supporting the

general approach of promoting cardiomyocyte renewal as a means to effect repair of injured hearts.

An interesting observation from the experiments reviewed here is that when cardiomyocyte renewal rates are similar to drop-out rates (as was seen in the atrial fibrosis, 
TAC and cardiotoxicity studies), the net effect is a marked reduction or absence of overt fibrosis. This suggests that the fibrosis normally observed in these injury models arise as a compensatory response to cardiomyocyte loss, as opposed to injury-induced activation of fibroblast signaling. Interestingly, this view is supported in cases where there is a massive acute loss of cardiomyocytes (as was seen in the coronary artery ligation model); although overt scar tissue persists, only minimal fibrosis is observed in the de novo myocardium. Persistence of an overt scar may reflect the need to retain wall integrity to prevent cardiac rupture at early stages post-injury. Although higher rates of cell cycle renewal may reduce persistent scar content, it may come at the cost of greater vulnerability to cardiac rupture. The potential contribution of a large number of proliferating cells to arrhythmia vulnerability must also be considered. Thus, it remains to be seen if slow vs. fast rates of cardiomyocyte renewal are ultimately better tolerated when effecting regenerative growth of the myocardium.

Another consideration for developing cardiomyocyte renewal-based approaches to effect cardiac regeneration is the likely need to incorporate cell-type specificity into the intervention. Most of the published studies rely on manipulating genetic pathways which can induce proliferation in a wide variety of cell types, including cardiomyocytes. It is possible that the used of vector systems with a tropism for targeting expression of cardiomyocytes will effect some degree of cell-type preference. Use of non-vector based approaches (as for example, delivery of RNA which has been modified for enhanced stability) may also be viable, provided a sufficient number of cardiomyocytes can be transduced. As work in the field progresses, it is possible that regulatory pathways which are intrinsic to and specific for cardiomyocyte renewal will be identified. Such pathways could likely be exploited to develop cell-type specific interventions. 
In conclusion, the studies reviewed here, as well as many other examples in the literature, make a strong case for the potential utility of inducing cardiomyocyte renewal as a means to treat injured hearts. Although a number of formidable obstacles must be overcome, advances in the field are accelerating and with it the hope of attaining a viable cardiomyocyte renewal-based intervention.

\section{Acknowledgment}

Arash Eghbali and Austin Dukes contributed equally to this work.

\section{Compliance with Ethical Standards}

\section{Conflict of Interest}

Arash Eghbali, Austin Dukes, Karl Toischer, and Loren J. Field declare that they have no conflict of interest.

Gerd Hasenfuss reports personal fees from Corvia, Servier, Impulse Dynamics, Novartis, AstraZeneca, Vifor Pharma, Berlin Chemie, and Springer.

\section{Human and Animal Rights and Informed Consent}

All reported studies/experiments with human or animal subjects performed by the authors have been previously published and complied with all applicable ethical standards (including the Helsinki declaration and its amendments, institutional/national research committee standards, and international/national/institutional guidelines).

\section{References}

Papers of particular interest, published recently, have been highlighted as: 


\section{- Of importance}

\section{•. Of major importance}

1. Ali SR, Hippenmeyer S, Saadat LV, Luo L, Weissman IL, Ardehali R. Existing cardiomyocytes generate cardiomyocytes at a low rate after birth in mice. Proc Natl Acad Sci U S A. 2014;111(24):8850-5. doi:10.1073/pnas.1408233111.

2. Senyo SE, Steinhauser ML, Pizzimenti CL, Yang VK, Cai L, Wang M et al. Mammalian heart renewal by preexisting cardiomyocytes. Nature. 2013;493(7432):433-6. doi:10.1038/nature11682.

3. • Eschenhagen T, Bolli R, Braun T, Field LJ, Fleischmann BK, Frisen J et al. Cardiomyocyte Regeneration: A Consensus Statement. Circulation. 2017;136(7):680-6. doi:10.1161/CIRCULATIONAHA.117.029343.

This consensus statement delineates the lack of critical data supporting a direct cardiomyogenic role for purported cardiac resident stem cells, and thus underscores the need to develop alternative strategies for promoting myocardial regeneration.

4. Madonna R, Van Laake LW, Davidson SM, Engel FB, Hausenloy DJ, Lecour S et al. Position Paper of the European Society of Cardiology Working Group Cellular Biology of the Heart: cell-based therapies for myocardial repair and regeneration in ischemic heart disease and heart failure. Eur Heart J. 2016;37(23):1789-98. doi:10.1093/eurheartj/ehw113.

5. Soonpaa MH, Field LJ. Assessment of cardiomyocyte DNA synthesis in normal and injured adult mouse hearts. Am J Physiol. 1997;272(1 Pt 2):H220-6.

6. Soonpaa MH, Field L. Survey of studies examining mammalian cardiomyocyte DNA synthesis. Circ Res. 1998;83(1):15-26.

7. Bergmann O, Bhardwaj RD, Bernard S, Zdunek S, Barnabe-Heider F, Walsh S et al. Evidence for cardiomyocyte renewal in humans. Science. 2009;324(5923):98-102. doi:10.1126/science.1164680.

8. Bergmann O, Zdunek S, Felker A, Salehpour M, Alkass K, Bernard S et al. Dynamics of Cell Generation and Turnover in the Human Heart. Cell. 2015;161(7):1566-75. doi:10.1016/j.cell.2015.05.026.

9. Swynghedauw B. Molecular mechanisms of myocardial remodeling. Physiol Rev. 1999;79(1):215-62.

10. Pasumarthi KB, Field LJ. Cardiomyocyte cell cycle regulation. Circ Res. 2002;90(10):1044-54.

11. Field L. Atrial natriuretic factor-SV40 T antigen transgenes produce tumors and cardiac arrhythmias in mice. Science. 1988;239(4843):1029-33.

12. Behringer RR, Peschon JJ, Messing A, Gartside CL, Hauschka SD, Palmiter RD et al. Heart and bone tumors in transgenic mice. Proc Natl Acad Sci U S A. 1988;85(8):2648-52.

13. Katz EB, Steinhelper ME, Delcarpio JB, Daud Al, Claycomb WC, Field LJ. Cardiomyocyte proliferation in mice expressing alpha-cardiac myosin heavy chain-SV40 T-antigen transgenes. Am J Physiol. 1992;262(6 Pt 2):H186776.

14. Pasumarthi KB, Nakajima H, Nakajima HO, Soonpaa MH, Field LJ. Targeted expression of cyclin D2 results in cardiomyocyte DNA synthesis and infarct regression in transgenic mice. Circ Res. 2005;96(1):110-8.

15. Hassink RJ, Pasumarthi KB, Nakajima H, Rubart M, Soonpaa MH, de la Riviere AB et al. Cardiomyocyte cell cycle activation improves cardiac function after myocardial infarction. Cardiovasc Res. 2008;78(1):18-25.

16. Nakajima H, Nakajima HO, Salcher O, Dittie AS, Dembowsky K, Jing S et al. Atrial but not ventricular fibrosis in mice expressing a mutant transforming growth factor-beta(1) transgene in the heart. Circ Res.

2000;86(5):571-9.

17. Nakajima H, Nakajima HO, Dembowsky K, Pasumarthi KB, Field LJ. Cardiomyocyte cell cycle activation ameliorates fibrosis in the atrium. Circ Res. 2006;98(1):141-8. 
18. - Toischer K, Zhu W, Hunlich M, Mohamed BA, Khadjeh S, Reuter SP et al. Cardiomyocyte proliferation prevents failure in pressure overload but not volume overload. J Clin Invest. 2017;127(12):4285-4296. doi:10.1172/JCl81870.

Findings from this study suggest that the presence of cardiomyocyte cell cycle activity can block the onset of heart failure resulting from chronic increases in cardiac after-load.

19. - Zhu W, Reuter S, Field LJ. Targeted expression of cyclin D2 ameliorates late stage anthracycline cardiotoxicity. Cardiovasc Res. 2019;115(5):960-5. doi:10.1093/cvr/cvy273.

Findings from this study demonstrate that the presence of cardiomyocyte cell cycle activity can reverse heart failure in a chronic juvenile doxorubicin cardiotoxicity model.

20. Zhu W, Shou W, Payne RM, Caldwell R, Field L. A mouse model for juvenile doxorubicin-induced cardiac dysfunction. Pediatric research. 2008;64(5):488-94.

\section{Figure Legends}

Figure 1. Targeted expression of cyclin D2 results in cardiomyocyte cell cycle activity which can promote structural and functional recovery following myocardial injury. A. Schematic diagram of the MHC-cycD2 transgene (upper panel), and documentation of transgene expression (lower left panel, anti-cyclin D2 immune histology, brown signal), endogenous CDK4 expression (lower middle panel, anti-CDK4 immune histology, brown signal) and cardiomyocyte S-phase activity (lower right panel, tritiated thymidine incorporation in cardiomyocyte nuclei, black punctate signal over blue nuclear signal). (Reproduced from:

Pasumarthi KB, Nakajima H, Nakajima HO, Soonpaa MH, Field LJ: Circ Res. 2005;96(1):1108, with permission from Wolters Kluwer Health, Inc.) [14] B. Histologic analysis of WT and D2 hearts at 7 and 180 days post-infarction, sections sampled at $1 \mathrm{~mm}$ intervals from apex to base and stained with Azan (magenta indicates viable myocardium, grey indicates scar). (Reproduced from: Hassink RJ, Pasumarthi KB, Nakajima H, Rubart M, Soonpaa MH, de la Riviere AB et al.: Cardiovasc Res. 2008;78(1):18-25., with permission from Oxford University

Press) [15] C. Cardiac function (as quantitated by $\mathrm{dP} / \mathrm{dt}_{\max } / \mathrm{EDV}$ ) in WT (open bars) and D2 (solid bars) at 7,60 and 180 days post-infarction; data is normalized to the percent activity 
observed in sham-operated littermates. (Reproduced from: Hassink RJ, Pasumarthi KB, Nakajima H, Rubart M, Soonpaa MH, de la Riviere AB et al.: Cardiovasc Res. 2008;78(1):1825., with permission from Oxford University Press) [15] D. Histologic analysis of right atria from 12 week old TGF (left panel) and TGF / D2 double transgenic (right panel) mice; sections were stained with Sirius Red and Fast Green (green indicates viable myocardium, red indicates collagen deposition). (Reproduced from: Nakajima H, Nakajima HO, Dembowsky K, Pasumarthi KB, Field LJ: Circ Res. 2006;98(1):141-8, with permission from Wolters Kluwer Health, Inc.) [17].

Figure 2. Cyclin D2 expression blocks progression to heart failure in a chronic pressure overload model. A. Survival of WT (blue trace) and D2 (red trace) mice following TAC surgery (black trace shows survival of sham operated animals). B. Fractional shortening (FS) of WT and D2 mice 10 weeks after sham or TAC surgery as measured by echocardiography. $\mathbf{C}$. Detection of p-H3 immune reactivity in cardiomyocyte nuclei (left panel, black punctate signal over blue nuclear signal) and quantitation of levels in D2 mice 10 weeks after sham or TAC surgery. D. Cardiomyocyte number determined from dispersed cell preparations of WT and D2 hearts 10 weeks after sham or TAC surgery. E. Activated caspase 3-positive cardiomyocytes per $\mathrm{mm}^{2}$ tissue in WT and D2 mice at 3 weeks after sham or TAC surgery. F. Histologic analysis of WT (left panel) and D2 (right panel) mice 10 weeks after TAC; sections were stained with Sirius Red and Fast Green (green indicates viable myocardium, red indicates collagen deposition). (Reproduced from: Toischer K, Zhu W, Hunlich M, Mohamed BA, Khadjeh S, Reuter SP et al.: J Clin Invest. 2017;127(12):4285-4296, with permission from American Society for Clinical Investigation) [18]. 
Figure 3. Cyclin D2 expression restores cardiac function in a juvenile anthracycline cardiotoxicity model. A. Cardiac function in saline-treated WT (black trace), saline-treated D2 (magenta), DOX-treated WT (red trace) and DOX-treated D2 (blue trace) mice at various time points in the study; vertical arrows indicate the time of saline or DOX injections. B. Quantitation of tritiated thymidine incorporation and activated caspase 3 immune reactivity in DOX-treated WT and D2 mice at the acute and late stages; results are plotted as events per $\mathrm{mm}^{2}$ to allow direct comparison of the results. C. Histologic analysis of acute and late stage WT and D2 hearts 13 weeks after DOX treatment; sections were stained with Sirius Red and Fast Green (green indicates viable myocardium, red indicates collagen deposition). (Reproduced from: Zhu W, Reuter S, Field LJ.: Cardiovasc Res. 2019;115(5):960-5, with permission from Oxford University Press) [19]. 
Figure 1.

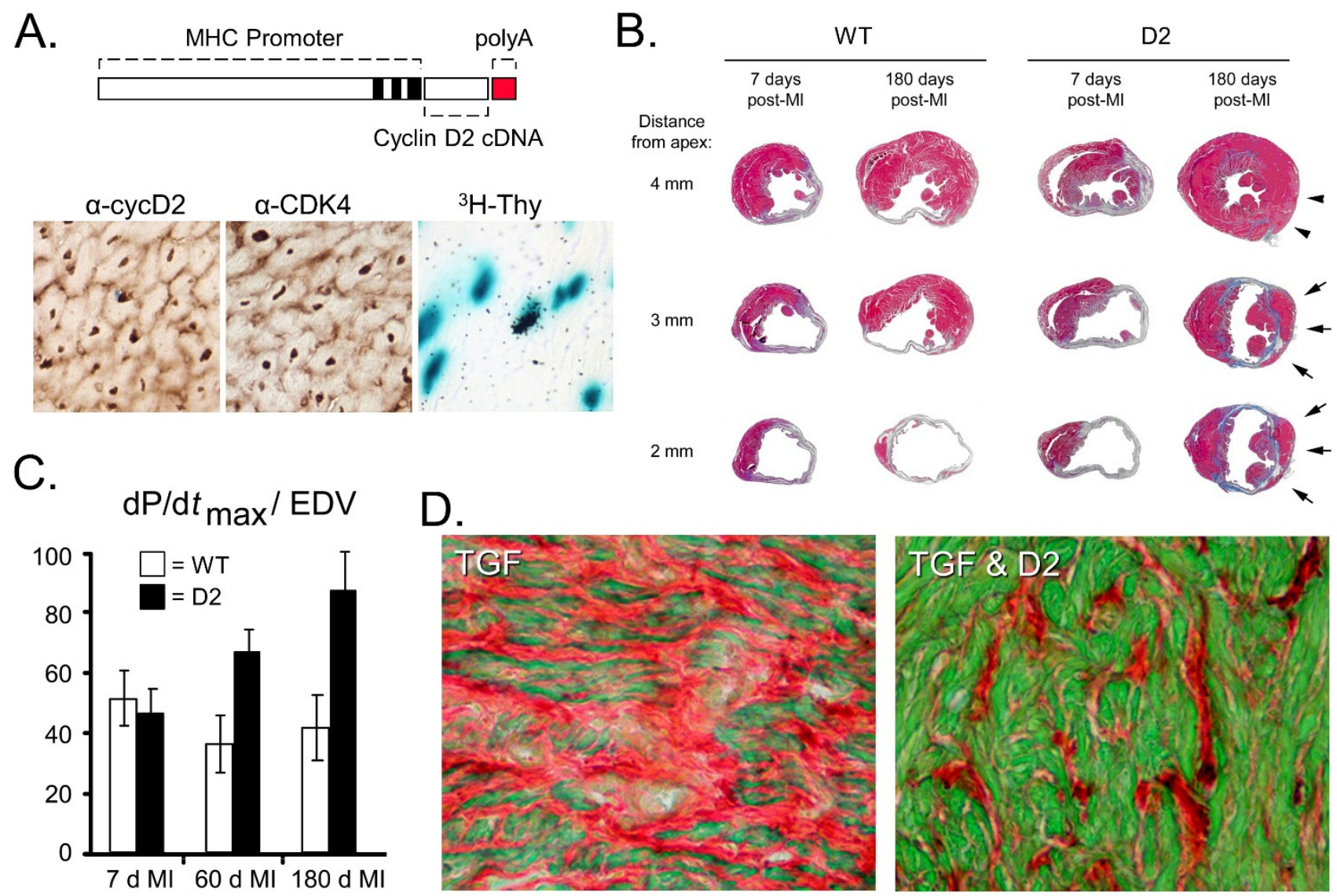


Figure 2.
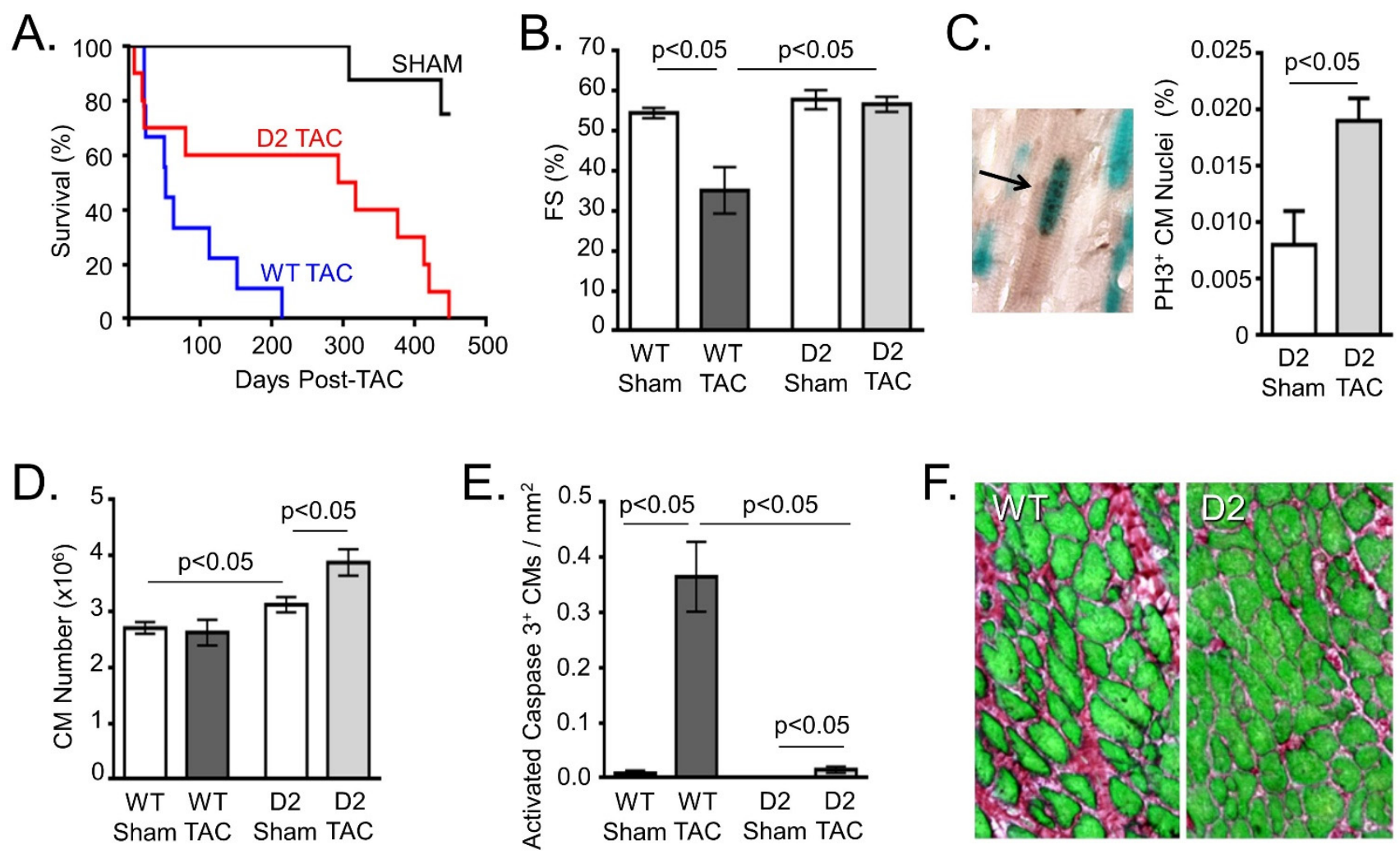
Figure 3 .

A

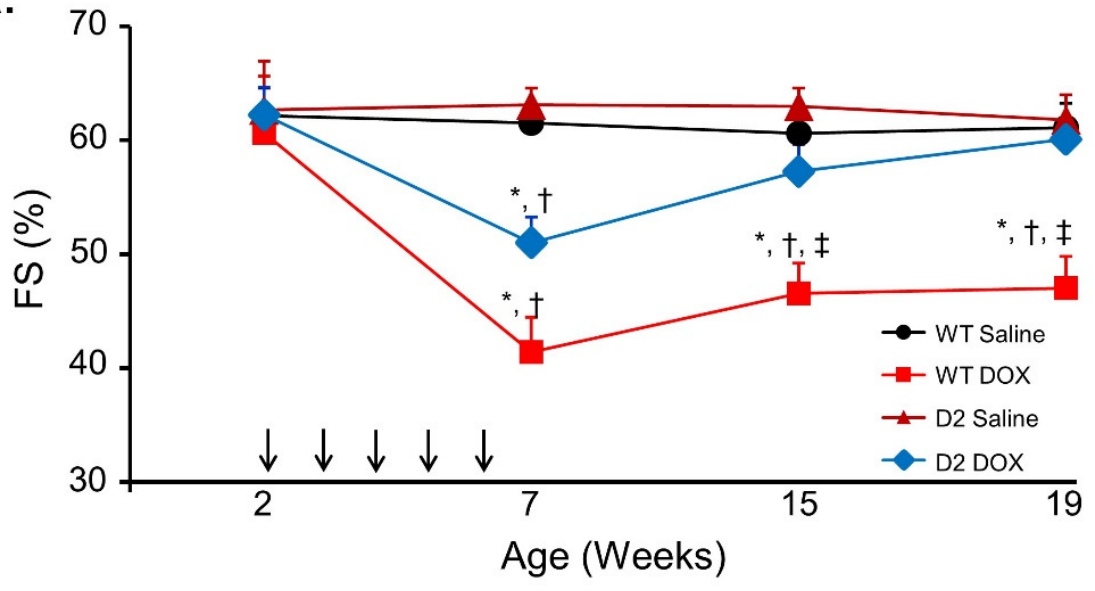

B.

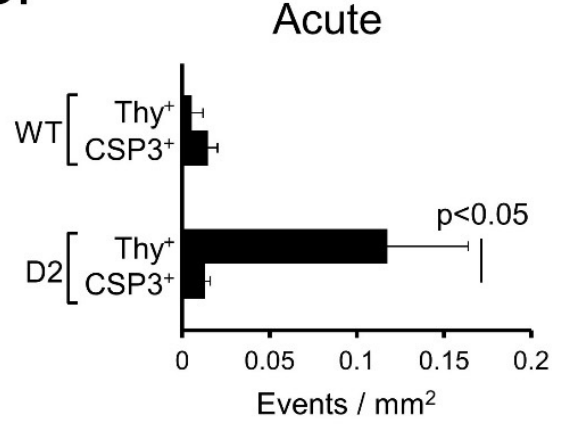

C. WTAcite
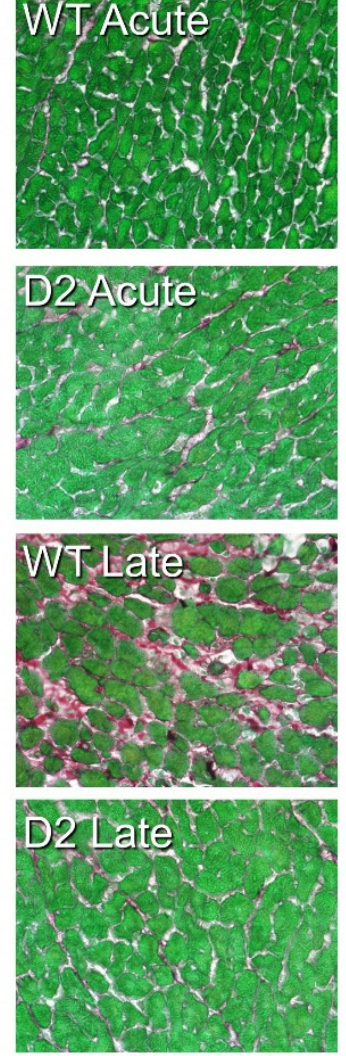